University for Business and Technology in Kosovo

UBT Knowledge Center

UBT International Conference

2013 UBT International Conference

Nov 2nd, 3:45 PM - 4:00 PM

\title{
Benchmarks for Hybrid Modelling
}

Andreas Koerner

Vienna University of Technology, andreas.koerner@tuwien.ac.at

Felix Breitenecker

Follow this and additional works at: https://knowledgecenter.ubt-uni.net/conference

Part of the Computer Sciences Commons, and the Engineering Commons

\section{Recommended Citation}

Koerner, Andreas and Breitenecker, Felix, "Benchmarks for Hybrid Modelling" (2013). UBT International Conference. 79.

https://knowledgecenter.ubt-uni.net/conference/2013/all-events/79

This Event is brought to you for free and open access by the Publication and Journals at UBT Knowledge Center. It has been accepted for inclusion in UBT International Conference by an authorized administrator of UBT Knowledge Center. For more information, please contact knowledge.center@ubt-uni.net. 


\title{
Benchmarks for Hybrid Modelling
}

\author{
Andreas Körner ${ }^{1}$ and Felix Breitenecker ${ }^{1}$ \\ Vienna University of Technology, Institute for Analysis and Scientific Computing, \\ Wiedner Hauptstrae 8-10, 1040 Vienna, Austria \\ andreas.koerner@tuwien.ac.at
}

\begin{abstract}
Hybrid Modelling is getting more and more important in technical and natural sciences. In these fields very complex systems and processes have to be simulated and therefore proper models must be developed. For complex systems different model structures for dynamical systems are available. One of the important model structures regarding complex dynamical systems are hybrid models. A hybrid model consists of several dynamical models and a state diagram where each state is described by a differential algebraic equation (DAE). This paper deals with benchmarks out of different fields of applications of this modelling approach. The first benchmark is an electrical circuit with linear devices and a diode as a nonlinear component. This example will show the influence of a simple model to the state space description or the corresponding differential algebraic description. The different models for the nonlinear behaviour of the diode influences the states of the state diagram. It is remarkable, that the different models for the diode results in a different hybrid model. The influence from these models on the diode can be observed. Another benchmark is the rotating pendulum. It is also described by a state diagram, in this special academic example a state diagram with two states. The two examples are representative, one out of the field of electrical engineering, one typical mechanical scenario. In the article two aspects will be considered. On the one side the modelling process of the electrical circuit will be observed in detail, especial the influence of different diode models on the structure of the states, and the state transition between the two states of the mechanical pendulum. On the other side also the need of a mathematical notation and description of the states and their transition will be discussed. In the end of the article some comments to the simulation of hybrid systems will be given.
\end{abstract}

Keywords: hybrid models, finite automaton, hybrid automaton, state-space models, benchmarks for simulation, modeling and simulation

\section{Introduction and Concept}

The paper mainly deals with benchmarks for hybrid modelling. This first section illustrates the organisation of the paper, gives a small introduction and explains the aims of the article. There are two main subjects who are focused in this paper: 
1. hybrid modelling approaches

2. benchmarks for simulation software regarding hybrid models

The following section will introduce the surroundings of hybrid modelling.

\section{$2 \quad$ Hybrid Modelling}

First of all the necessary definitions as in [1] will be given.

\subsection{Continuous-Time State-Space model}

Let $x \in \mathbb{R}^{n}$ denote the state variables and $w \in \mathbb{R}^{q}$ the external variables related by a mixed set of differential and algebraic equations of the form

$$
F(x, \dot{x}, w)=0 .
$$

Solutions of (1) are all sufficient smooth time functions $x$ and $w$ which are satisfying (1). In technical context the definition is adapted to an continuoustime input-state-output system of the form

$$
\begin{aligned}
& \dot{x}=f(x, u), \\
& y=h(x, u) .
\end{aligned}
$$

Therefor the vector $w$ is splited into a subvector $u \in \mathbb{R}^{m}$ and a subvector $y \in \mathbb{R}^{p}$, restricted by the condition $m+p=q . u$ is called the input vector or input variables and $y$ the output vector or the output variables. In representation (2) the differential part is related to the state and input variables, the algebraic part to state, input and output variables (see Fig. 1). In representation (1) are more additional algebraic constraints on the state space variables $x$.

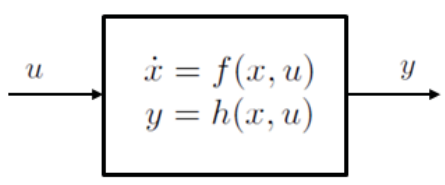

Fig. 1. Representation of a continuous-time input-state-output system

\section{$2.2 \quad$ Finite Automata}

A finite automaton is described by a triple $(L, A, E) . L$ is a finite set called the state space, $A$ is a finite set called the alphabet whose elements are called symbols. $E$ is the transition rule represented as a subset of $L \times A \times L$ and its elements are 


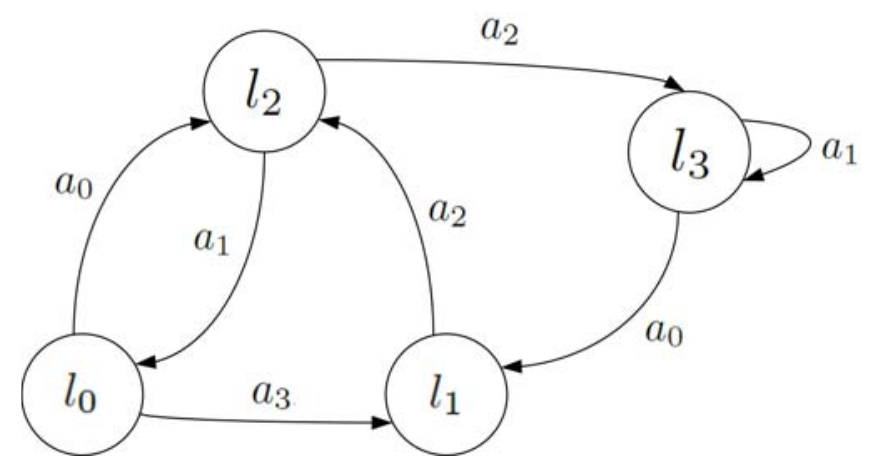

Fig. 2. Example of a finite automaton

called edges, transitions or events. A sequence $\left(l_{0}, a_{0}, l_{1}, a_{1}, \ldots, l_{n-1}, a_{n-1}, l_{n}\right)$ with $\left(l_{i}, a_{i}, l_{i+1}\right) \in E$, for $i=1,2, \ldots, n-1$, is called a trajectory or path.

Typical some finite automata are represented by a finite graph. A certain example of a finite automaton is shown in Fig. 2. Therefor the set of states consists of $L=\left\{l_{0}, l_{1}, l_{2}, l_{3}\right\}$ and the alphabet $A=\left\{a_{0}, a_{1}, a_{2}, a_{3}\right\}$. A can be interpreted as label on the edges of the graph and the elements of $L$ are denoting the nodes.

Also in context of finite automata there can be devepoped an input-output automaton. Therefor two symbols, namely an input symbol $i$ and an output symbol $o$, has to be choosen in the finite automaton. Deterministic input-output automata can be represented by eqations

$$
\begin{aligned}
l^{\sharp} & =\nu(l, i), \\
o & =\eta(l, i) .
\end{aligned}
$$

The symbol $l^{\sharp}$ in equation (3) denotes the new value of the state after the event takes place, resulting from the old discrete state value $l$ and the input $i$. An alternative formulation in this situation could be given: If $t_{k}$ is the time step before the event takes place and $t_{k+1}$ the time step after the event the relations for the output state can be considered as $l=l\left(t_{k}\right)$ and $l^{\sharp}=l\left(t_{k+1}\right)$. Thereby $l: \mathbb{Z} \rightarrow L$ is the time evolution of $l$.

Often the definition of a finite automaton includes the explicit specification of a subset $I \subset L$ of initial states and a subset $F \subset L$ of final states. A path $\left(l_{0}, a_{0}, l_{1}, a_{1}, \ldots, l_{n-1}, a_{n-1}, l_{n}\right)$ is called a successful path if the condition $l_{0} \in I$ and $l_{n} \in F$ is added.

In contrast to the continuous-time system the solution concept of a finite automaton with or without initial and final states is completely specified because the behavior of the finite automaton consists of all paths respectively successful paths. 


\subsection{Hybrid Automata}

The combination of the previous given definition of a continuous-time statespace system and a finite automaton leads to the definition of a hybrid automaton. A hybrid automaton is described by a tuple ( $L, X, A, W, E$, Inv, Act). Each of the Elements is defined as follows:

- $L$ is a finite set, called the set of discrete states.

- $X$ is the continuous state space of the hybrid automaton in which the continuous state variables $x$ take their values. Typically $X$ fulfills in applications $X \subset \mathbb{R}^{n}$, but also $n$-dimensional manifolds are imaginable.

- $A$ is a finite set of symbols which labels the nodes.

- $W=\mathbb{R}^{q}$ is the continuous communication space in which the continuous external variables $w$ take their values.

- $E$ is a finite set of edges, called transitions or events. Every edge is defined by a tuple $\left(l, a, \operatorname{Guard}_{l, l^{\prime}}, \operatorname{Jump}_{l, l^{\prime}}, l^{\prime}\right)$, where $l, l^{\prime} \in L, a \in A$. $\operatorname{Guard}_{l, l^{\prime}}$ is a subset of $X$ and Jumpl $l, l^{\prime}$ is a relation defined by a subset of $X \times X$. The transition from the discrete state $l$ to $l^{\prime}$ is called enabled, when the continuous state fulfills $x \in \operatorname{Guard}_{l, l^{\prime}}$. The continuous state $x$ jumps to a value $x^{\prime}$ if $\left(x, x^{\prime}\right) \in \operatorname{Jump}_{l, l^{\prime}}$.

- Inv is a mapping from the locations $L$ to the set of subsets of $X$ so that $\operatorname{Inv}(l) \subset X, \forall l \in L$. Whenever the system is at location $l$, the continuous state $x$ must satisfy $x \in \operatorname{Inv}(l) \operatorname{Inv}(l)$ for $l \in L$ is called the location invariant of location $l$.

- Act is a mapping that assigns to each location $l \in L$ a set of differentialalgebraic equations $F_{l}$, relating the continuous state variables $x$ with their time-derivatives $\dot{x}$ and the continuous external variables $w$ by

$$
F_{l}(x, \dot{x}, w)=0 .
$$

The solution of these differential-algebraic equations are called the activities of the location $l$.

The illustration of a certain example is displayed in Fig. 3. Each node represents a state and a corresponding DAE system. For the transition $\left(l_{0}, a_{3}, l_{1}\right)$ the sets Guard and Jump is also illustrated.

A continuous trajectory $(l, \delta, x, w)$ associated with a location $l$ consists of a nonnegative time $\delta$, the duration of the continuous trajectory, a piecewise continuous function $w:[0, \delta] \rightarrow W$ and a continuous and piecewise differentiable function $x:[0, \delta] \rightarrow X$ which fulfill

1. $x(t) \in \operatorname{Inv}(l)$ for all $t \in(0, \delta)$,

2. $F_{l}(x(t), \dot{x}(t), w(t))=0$ for all $t \in(0, \delta)$ except for points of discontinuity of $w$.

A trajectory of the hybrid automaton is an (infinite) sequence of continuous trajectories

$$
\left(l_{0}, \delta_{0}, x_{0}, w_{0}\right) \stackrel{a_{0}}{\rightarrow}\left(l_{1}, \delta_{1}, x_{1}, w_{1}\right) \stackrel{a_{7}}{\rightarrow}\left(l_{2}, \delta_{2}, x_{2}, w_{2}\right) \stackrel{a_{2}}{\rightarrow} \ldots
$$




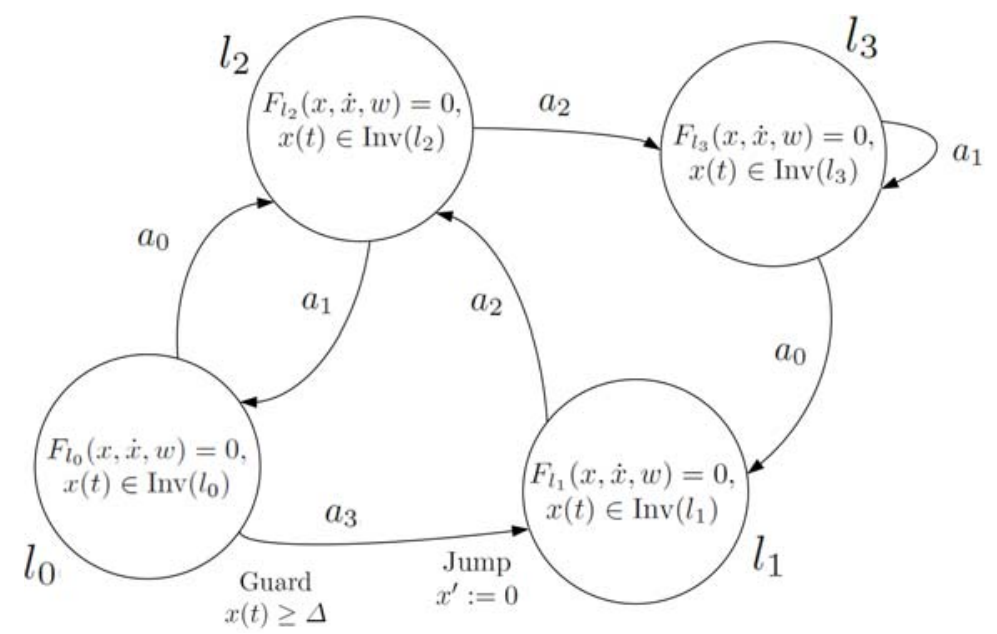

Fig. 3. Example of a hybrid automaton

such that at the event times

$$
t_{0}=\delta_{0}, \quad t_{1}=\delta_{0}+\delta_{1}, \quad t_{2}=\delta_{0}+\delta_{1}+\delta_{2}, \quad \ldots,
$$

the following inclusions hold for the discrete transitions

$$
\begin{gathered}
x_{j}\left(t_{j}\right) \in \operatorname{Guard}_{l_{j}, l_{j+1}}, \\
\left(x_{j}\left(t_{j}\right), x_{j+1}\left(t_{j}\right)\right) \in \operatorname{Jump}_{l_{j}, l_{j+1}}
\end{gathered}
$$

for all $j=0,1,2, \ldots$

\section{Benchmarks}

This section is about the two academic benchmarks to apply the theory of hybrid systems introduced in the last section. The idea of ARGESIM benchmarks [2] is to use the hybrid modelling approach to create models for technical systems or processes out of natural sciences. For this reason two Benchmarks are introduced:

1. hybrid electrical circuit

2. rotating pendulum

The benchmarks work on several case studies which are defined to find out the potential and constraints of different simulation software. Also the ability to work with common mathematical model description is included in the focus.

The following subsections will show the way to model such hybrid system descriptions. 


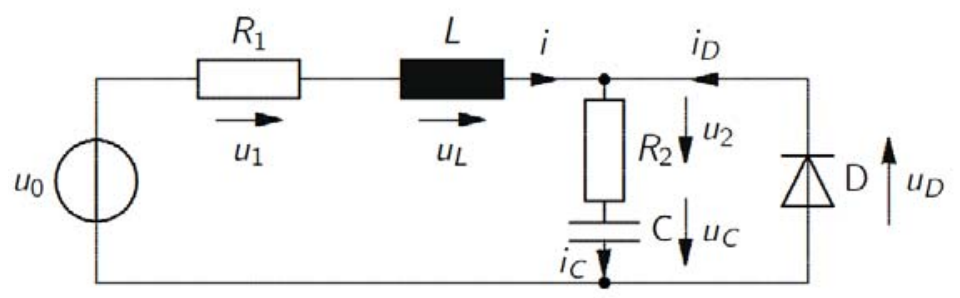

Fig. 4. Benchmark hybrid electrical circuit

\subsection{Hybrid Electrical Circuit}

The observed circuit is an ordinary series resonant circuit combined with a diode. The circuit is illustrated in Fig. 4.

To find the whole description of the circuit the different sates have to be defined. Therefor the two states of the diode have to be considered. The used model of the diode is given by the relation of voltage $u_{D}$ and current $i_{D}$ of the diode. The link is given by the so called Shockley-equation

$$
i_{D}\left(u_{D}\right)= \begin{cases}I_{S}\left(\mathrm{e}^{\frac{u_{D}}{U_{T}}}-1\right) & \text { for } u_{D}>0 \\ 0 & \text { for } u_{D} \leq 0 .\end{cases}
$$

The Parameter $I_{S}, U_{T}>0$ are characteristic for the diode and the influence can be investigated by several parameter studies. For the modelling process we will not focus on these parameter studies. The two states of the diode lead to observe two different subsystems which will be combined in the whole hybrid system.

The first subsystem is represented by a series resonant circuit. The equations for the inductance $L$ and the capacity $C$

$$
u_{L}(t)=L \frac{\mathrm{d} i}{\mathrm{dt}} \quad \text { and } \quad i(t)=C \frac{\mathrm{d} u_{C}}{\mathrm{dt}}
$$

according to the Kirchhoff law of voltages

$$
u_{1}(t)+u_{L}(t)+u_{2}(t)+u_{C}(t)=u_{0}(t)
$$

results the two equations

$$
\frac{\mathrm{d}}{\mathrm{dt}} u_{C}(t)=\frac{1}{C} i(t), \quad \frac{\mathrm{d}}{\mathrm{dt}} i(t)=-\frac{1}{L} u_{C}(t)-\frac{R_{1}+R_{2}}{L} i(t)+\frac{1}{L} u_{0}(t) .
$$

A sketch of the circuit for this subsystem and the quantity to be measured is illustrated in Fig. 5.

The state-space description is known as

$$
\dot{x}=A x+B u,
$$




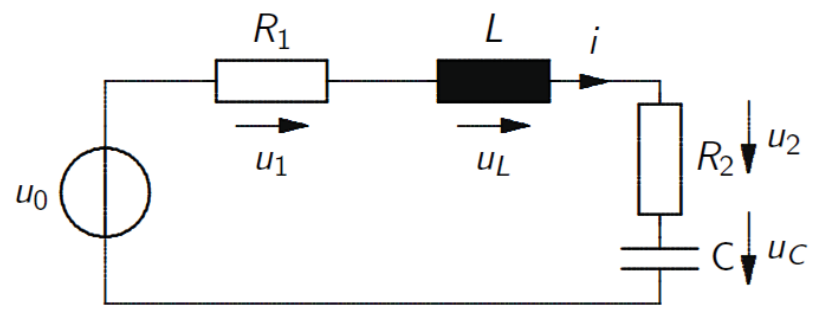

Fig. 5. Subsystem 1 of the hybrid electrical circuit

where $x \in \mathbb{R}^{n}, u \in \mathbb{R}^{d}$ as well as $A \in \mathbb{R}^{n \times n}$ and $B \in \mathbb{R}^{n \times d}$. For the considered series resonant circuit follows from the above

$$
\frac{\mathrm{d}}{\mathrm{dt}}\left(\begin{array}{c}
u_{C}(t) \\
i(t)
\end{array}\right)=\left(\begin{array}{cc}
0 & \frac{1}{C} \\
-\frac{1}{L}-\frac{R_{1}+R_{2}}{L}
\end{array}\right)\left(\begin{array}{c}
u_{C}(t) \\
i(t)
\end{array}\right)+\left(\begin{array}{l}
0 \\
\frac{1}{L}
\end{array}\right) u_{0}(t) .
$$

For the second state of the hybrid electrical circuit the ordinary system can be adapted without doing the modelling process again from the beginning. The subsystem 2 is not really a subsystem but the first subsystem can be used for formulating the model equation as follows. The equations for the inductance $L$ and the capacity $C$ according to the Kirchhoff law of currents and voltages

$$
i(t)+i_{D}(t)=i_{C}(t) \quad \text { and } \quad u_{1}(t)+u_{L}(t)+u_{2}(t)+u_{C}(t)=u_{0}(t)
$$

results the equations

$$
\frac{\mathrm{d}}{\mathrm{dt}} u_{C}(t)=\frac{1}{C} i(t)+\frac{1}{C} i_{D}(t)
$$

and

$$
\frac{\mathrm{d}}{\mathrm{dt}} i(t)=-\frac{1}{L} u_{C}(t)-\frac{R_{1}+R_{2}}{L} i(t)+\frac{1}{L} u_{0}(t)-\frac{R_{2}}{L} i_{D}(t) .
$$

This equations lead to the description of the hybrid electrical circuit for the state $u_{D}>0$ as

$$
\frac{\mathrm{d}}{\mathrm{dt}}\left(\begin{array}{c}
u_{C}(t) \\
i(t)
\end{array}\right)=\left(\begin{array}{cc}
0 & \frac{1}{C} \\
-\frac{1}{L}-\frac{R_{1}+R_{2}}{L}
\end{array}\right)\left(\begin{array}{c}
u_{C}(t) \\
i(t)
\end{array}\right)+\left(\begin{array}{c}
0 \\
\frac{1}{L}
\end{array}\right) u_{0}(t)+\left(\begin{array}{c}
\frac{1}{C} \\
-\frac{R_{2}}{L}
\end{array}\right) i_{D}(t) .
$$

But this (nonlinear) state-space description is not enough because in the ordinary differential equation appears the algebraic variable $u_{D}$. Therefor an additional equation is needed. Together with equation(8) and the Kirchhoff law for voltages in the second loop the algebraic equation for $u_{D}$ can be derived as

$$
u_{2}(t)+u_{C}(t)+u_{D}(t)=0 .
$$

The condition $u_{D}>0$ is equivalent to $u_{2}+u_{C}>0$ and the algebraic equation is given by

$$
R_{2} i(t)+u_{c}(t)+R_{2} I_{S}\left(\mathrm{e}^{\frac{u_{D}}{U_{T}}}-1\right)+u_{D}=0
$$


This algebraic equation is of the form

$$
g(x, z, p)=0
$$

where $x \in \mathbb{R}^{n}$ is the state vector, $z \in \mathbb{R}^{r}$ is the vector of the algebraic variables and $p \in \mathbb{R}^{q}$ a parameter vector if necessary. For this academic example apply $z=u_{D}$ and $p=\left(R_{1}, R_{2}, C, L, I_{S}, U_{T}\right)^{T}$.

The condition $u_{D}>0$, or equivalent $u_{2}+u_{C}>0$ defines two states of the hybrid electrical circuit. The corresponding graph for the hybrid automaton is shown in Fig. 6.

$$
\begin{aligned}
& \begin{array}{c}
\frac{\mathrm{d}}{\mathrm{dt}}\left(\begin{array}{c}
u_{C}(t) \\
i(t)
\end{array}\right)=\left(\begin{array}{cc}
0 & \frac{1}{C} \\
-\frac{1}{L} & -\frac{R_{1}+R_{2}}{L}
\end{array}\right)\left(\begin{array}{c}
u_{C}(t) \\
i(t)
\end{array}\right)+\left(\begin{array}{l}
0 \\
\frac{1}{L}
\end{array}\right) u_{0}(t) \\
\substack{\mathrm{L}>0 \\
u_{D}>0} \\
\quad
\end{array} \\
& \begin{array}{l}
\frac{\mathrm{d}}{\mathrm{dt}}\left(\begin{array}{c}
u_{C}(t) \\
i(t)
\end{array}\right)=\left(\begin{array}{cc}
0 & \frac{1}{C} \\
-\frac{1}{L} & -\frac{R_{1}+R_{2}}{L}
\end{array}\right)\left(\begin{array}{c}
u_{C}(t) \\
i(t)
\end{array}\right)+\left(\begin{array}{l}
0 \\
\frac{1}{L}
\end{array}\right) u_{0}(t)+\left(\begin{array}{c}
\frac{1}{C^{2}} \\
-\frac{R_{2}}{L}
\end{array}\right) i_{D}(t) \\
R_{2} i(t)+u_{C}(t)+R_{2} I_{S}\left(\mathrm{e}^{\frac{u_{D}}{U_{T}}}-1\right)+u_{D}=0
\end{array}
\end{aligned}
$$

Fig. 6. Graph of the hybrid automaton of the hybrid electrical circuit

\subsection{Rotating Pendulum}

The second considered example is the rotating pendulum. On the basis of the definition given in [3] the benchmark deals with a mass working as a pendulum on the one hand side and as a mass in the gravity field on the other hand side. The geometrical relation and the two states are shown in Fig. 7.
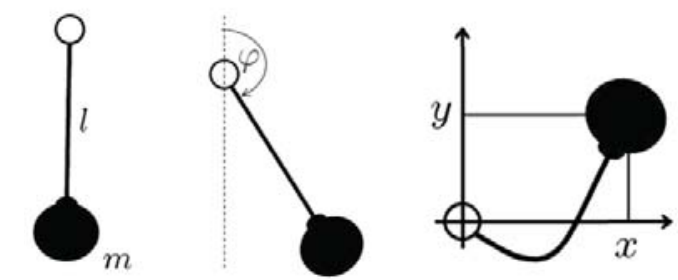

Fig. 7. Illustration of the geometry and the possible states of the rotating pendulum 
The equations for the description of the two states of the pendulum is the outcome of first principal modelling, as introduced in [4], in the two scenarios. This scenario is quite different to the electrical circuit with diode, because the two states of the rotating pendulum use different coordinate systems. The movement on the trajectory of a pendulum with tight rope is described in polar coordinates by the differential algebraic equations

$$
\begin{gathered}
\ddot{\varphi}+\frac{k}{m} \dot{\varphi}-\frac{g}{m} \sin \varphi=0, \\
F=-m g \cos \varphi+m l \dot{\varphi}^{2} .
\end{gathered}
$$

Thereby $\varphi$ is the polar angle and $(k, m, g, l)^{T}$ represents the parameter vector of this system. The second state of the rotating pendulum is defined for the case that the rope is no longer tight. Therefor the mass of the pendulum acts like a punctiform mass in the gravitation field. The common description for this process is formulated in Cartesian coordinates by the differential equations

$$
\begin{gathered}
m \ddot{x}=-k \dot{x}, \\
m \ddot{y}=-m g-k \dot{y} .
\end{gathered}
$$

The interesting point of view in this situation is to distinguish the two states. This cannot be done via one condition as in the case of the electrical circuit, in the case of the rotating pendulum one condition defines the transition from the state "circular movement" to "free fall" and another condition describes the transition backwards. For the hybrid model of the rotating pendulum a graph representing the states and their transitions is shown in Fig. 8. In this graph also the transformation of coordinates is denotet as well as the state variables in each nodes to focuses the size of the state space.

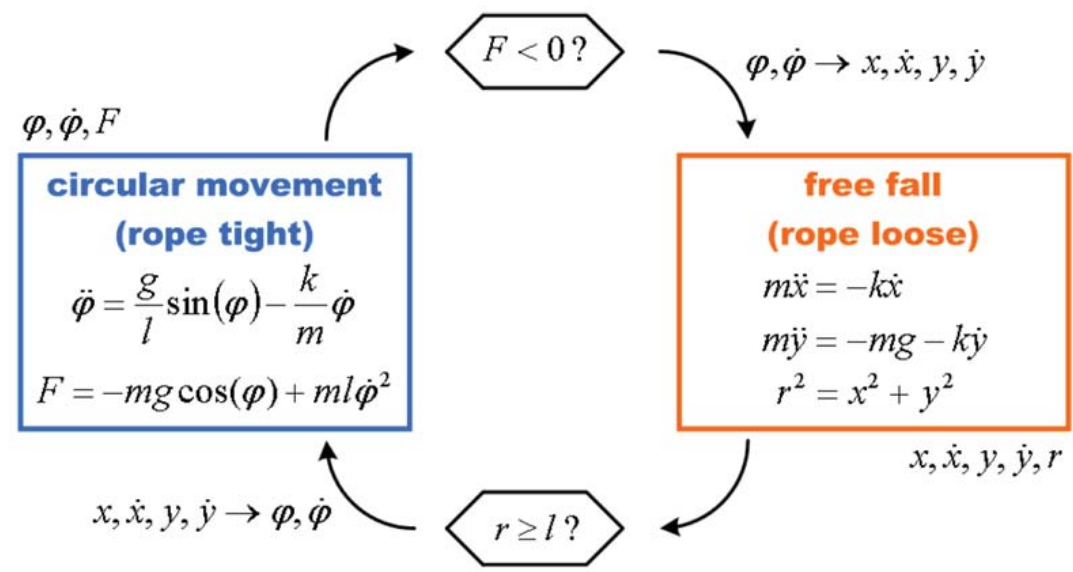

Fig. 8. Graph of representing the two states of the rotating pendulum 


\section{Summary and Outlook}

In section 3 the modelling process of the two academic examples - hybrid electrical circuit and rotating pendulum - were presented. The next step is to create out of these models several tasks for simulation software. As mentioned in the introduction the model will be used for benchmarking different simulation software to outline their pros and cons. To focus on several minimum abilities of simulation software some common tasks will be formulated like the calculation of eigenvalues of some subsystems in different scenarios, or the implementation of the differential and algebraic equations with different initial values. In the benchmark of the rotating pendulum special attention has to be given to the transformation on coordinates. Some simulators are not able to transform coordinates and so the users solve this problem by calculating the representations in both simulators which is not appropriate.

The benchmarks focus also on some numerical considerations. One example is the condition of the Jacobian matrix. In this context the addressed Jacobian matrix is formulated as follows. The description of a certain state is formulated through the introduced equation (1). This represents a equation for the differential variables but also the algebraic variables are covered. So the Jacobian matrix in this context is simplified to a gradient and the singular points are derived by

$$
\operatorname{grad} F=0 .
$$

Several tasks will be formulated for the benchmarks, also focusing on the modelling approach. The resulting benchmark emphasis on the ability of simulating hybrid systems. The final definition of the benchmark will be offer several assignments and will be published on [2].

Further work will be to adapt these models in this way that the model approaches offer the possibility for a wider range of simulation software, for example block or graphic oriented simulation software.

\section{References}

[1] van der Schaft A.J., Schumacher J.M.: An Introduction to Hybrid Dynamical Systems, Springer, 2007

[2] ARGESIM Benchmarks, www.argesim.org

[3] Körner A., Heinzl B., Rößler M., Winkler S., et al: BCP - A Benchmark For Hybrid Modelling and State Event Modelling, EUROSIM 2010 - 7th Congress on Modelling and Simulation, Prague, Czech Republic, Proceedings of the 7th Congress on Modelling and Simulation, Snorek M., Buk Z., Cepek M., Drchal J. (Edt.), Vol.2 Full Papers (2010), ISBN: 978-80-01-04589-3; p. 1032 - 1042

[4] Eck G., Garcke H., Knabner P.: Mathematische Modellierung, Springer, 2010 